\title{
Eustachian Valve Endocarditis
}

\author{
Viviane Cordeiro Veiga, Ana Cristina Carlo Magno Molinari, Cíntia Mara Farias, Amilton Silva Junior, Elias César Hauy \\ Marum, Salomón Ordinola Rojas, Marcelo Luiz Patrício, Henry Abensur \\ Real e Benemérita Sociedade Portuguesa de Beneficência - Sao Paulo, SP, Brazil
}

Eustachian valve endocarditis is a rare condition that affects mostly injectable drug users and patients using pacemakers or central venous catheter. We describe the case of a patient who underwent myocardial revascularization with extracorporeal circulation and who presented Eustachian valve endocarditis in the postoperative period, diagnosed by echocardiogram. The blood culture identified Klebsiella pneumoniae. He received appropriate antibiotic therapy and presented resolution of the condition within four weeks.

\section{Introduction}

Eustachian valve endocarditis is a rare condition that affects mostly injectable drug users and patients using pacemakers or central venous catheter ${ }^{1,2}$.

In fetal circulation, the Eustachian valve deviates blood from the inferior vena cava to the left atrium through the oval foramen.

The objective of this study is to report a case of Eustachian valve endocarditis in the postoperative period of cardiac surgery.

\section{Case Report}

A 45-year-old male patient, with diagnosis of coronary insufficiency, who underwent myocardial revascularization with anastomosis from the right internal thoracic artery to the right coronary artery, as well as from the left radial artery to the left marginal branch, with extracorporeal circulation and no intercurrent events. The invasive procedures during surgery were mean arterial pressure and placement of a central venous catheter. The patient was discharged from the intensive care unit on the first postoperative day in good clinical conditions, on prophylactic antibiotic therapy with cefuroxime $750 \mathrm{mg}$, three times a day.

On the third postoperative day, he presented abdominal bloating and on the next day, shivering and fever. Blood was withdrawn for culture, the central venous catheter was removed and the patient initiated on empirical antibiotic therapy with ciprofloxacin 400mg/day.

\section{Key words}

Endocarditis; Eustachian valve, heart valves.

Mailing address: Viviane Cordeiro Veiga •

Alameda Hungria, 89 - 06474-140 - Barueri, SP

E-mail: vcveiga@cardiol.br

Manuscript received May 19, 2006; revised manuscript received August 16, 2006; Accepted September 14, 2006.
The results of blood culture and microbiological examination of the central venous line tip was Klebsiella pneumoniae resistant to ciprofloxacin and sensitive to imipenem, which was prescribed at $2 \mathrm{~g} /$ day, with clinical and laboratory improvement.

A transthoracic Doppler echocardiogram was performed to investigate endocarditis and the following were observed: preserved right and left ventricular function, discreet mitral and tricuspid valve reflux and presence of a mobile mass on the right atrium.

Therefore, a transesophageal echocardiogram was required and showed a mobile and hyperechogenic mass on the right atrium, with random movement and measuring approximately $30 \mathrm{~mm}$, attached to the Eustachian valve, suggesting an endocardial vegetation (Figure 1).

The infectious condition improved and the patient had no fever as of the $5^{\text {th }}$ day on antibiotic, with simultaneous improvement in complete blood count.

On day 26 of specific therapy, a control transesophageal echocardiogram was performed and the Eustachian valve was observed with no vegetation signs. No other alterations were found in the echocardiogram. The patient was afebrile and the laboratory test results were not compatible with an infectious condition.

He was discharged after four-week antibiotic therapy, with no clinical or laboratory signs of infection (Figure 2).

\section{DISCUSSION}

Eustachian valve endocarditis is a rare condition and there

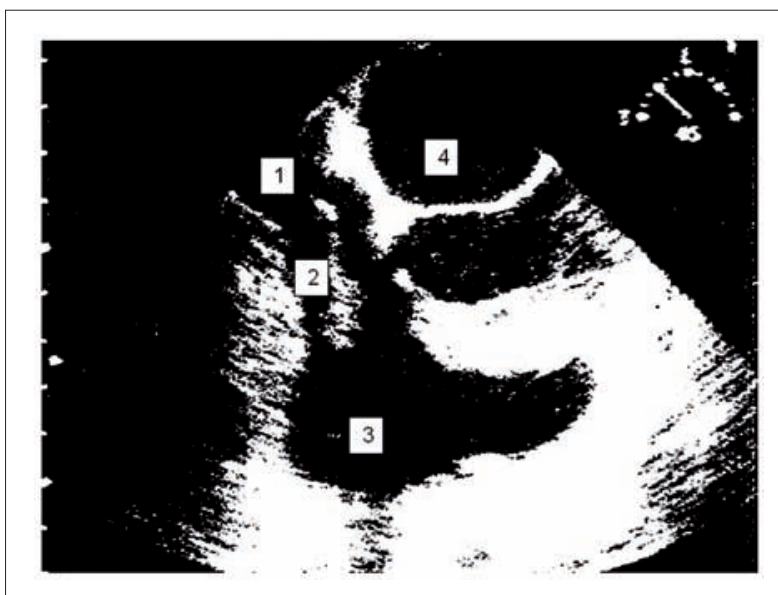

Fig. 1 - Presence of vegetation in Eustachian valve visualized on transesophageal echocardiogram. 1. Inferior vena cava; 2 . Eustachian valve with vegetation; 3. Right atrium; 4. Left atrium. 


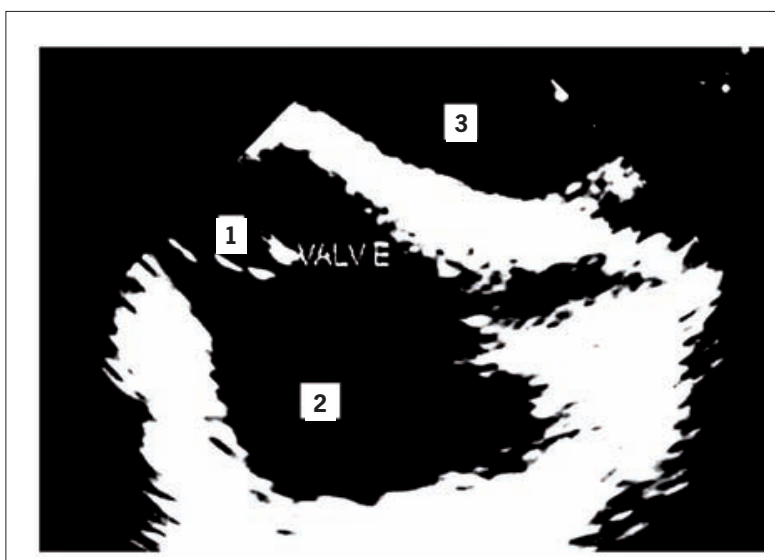

Fig. 2 - Transesophageal echocardiogram performed after four-week antibiotic therapy, showing the Eustachian valve with no signs of endocarditis. 1. Eustachian valve; 2. Right atrium; 3. Left atrium.

are few cases described in the literature ${ }^{1,3}$, which are usually associated with tricuspid valve endocarditis ${ }^{4}$.

The most common predisposing factor for this disease is use of injectable drugs; it is also related to placement of central venous catheter and use of pacemakers ${ }^{5,6}$. In the case reported,

\section{References}

1. James PR, Dawson D, Hardman SM. Eustachian valve endocarditis diagnosed by transesophageal echocardiography. Heart. 1999; 81: 91.

2. Palakodeti V, Keen WD, Rickman LS, Blanchard DG. Eustachian valve endocarditis detection with multiplane transesophageal echocardiography. Clin Cardiol. 1997; 20 (6): 579-80.

3. Villacosta I, San Roman JA, Roca V. Eustachian valve endocarditis. Br Heart J. 1990; 64: 340-1.

4. San Roman JA, Villacosta I, Sarria C, Garcimartin I, Rollan MJ, FernandezAviles F. Eustachian valve endocarditis: is it worth searching for? Am Heart J. 2001; 142: 1037-40.

5. Pellicelli AM, Pino P, Terranova A, Ambrosio C, Soccorsi F. Eustachian valve endocarditis: a rare localization of right side endocarditis. A case report and review of the literature. Cardiovasc Ultrasound. 2005; 3: 30.
Eustachian valve endocarditis resulted from a central venous catheter placed during a myocardial revascularization surgery.

The agent more often isolated in this condition is Staphylococus aureus ${ }^{5,7,8}$, and there are no reports of Klebsiella pneumoniae, as observed in this patient.

The diagnosis of Eustachian valve endocarditis is commonly made by means of transesophageal echocardiogram, which enables better visualization of the region as compared with transthoracic echocardiogram. Nevertheless, the latter remains the first choice exam; the transesophageal echocardiogram should be carried out if any vegetation is suspected on a transthoracic echocardiogram, or in case of a clinical picture suggesting endocarditis ${ }^{5,9,10}$.

The initial treatment for this condition is clinical, with appropriate antibiotic therapy, and surgery is indicated in non-responsive cases. Our patient presented remission of the infectious process after four-week treatment with specific antibiotics and required no surgical intervention.

\section{Conclusion}

Isolated Eustachian valve endocarditis is a condition that should be investigated in case of clinical suspicion in patients using central venous catheters.

6. Bowers J, Krimsky W, Gradon JD. The pitfalls of transthoracic echocardiography. A case of eustachian valve endocarditis. Tex Heart Inst J. 2001; 28: 57-9.

7. Schmidt MA, Nigbor D, Eitzman DT. Eustachian valve endocarditis caused by Streptococcus viridans. J Am Soc Echocardiogr. 2001; 14: 1042-3.

8. Pintor E, Gómez C, González J, Fernández-Cruz A, Almería C, Zamorano J. Bacteriemia por Staphylococcus aureus y masa en la aurícula derecha en un paciente portador de una via central. Rev Esp Cardiol. 1998;51:158-60.

9. Sawhney N, Palakodeti V, Raisinghani A, Rickman LS, De Maria NA, Blanchard DG. Eustachian valve endocarditis: a case series and analysis of the literature. J Am Soc Echocardiogr. 2001; 14: 1139-42.

10. Edwards AD, Vickens MA, Morgan CJ. Infective endocarditis affecting the Eustachian valve. Br Heart J. 1986; 56: 561-2. 